\title{
A deep narrowband survey for planetary nebulae at the outskirts of $M 3^{\star}$
}

\author{
R. Galera-Rosillo ${ }^{1,2}$, R. L. M. Corradi ${ }^{1,2,3}$, and A. Mampaso ${ }^{1,2}$ \\ ${ }^{1}$ Instituto de Astrofísica de Canarias, Calle Vía Láctea, s/n, 38205 La Laguna, Tenerife, Spain \\ e-mail: rgr@iac.es \\ 2 Departamento de Astrofísica, Universidad de La Laguna, 38206 La Laguna, Tenerife, Spain \\ ${ }^{3}$ GRANTECAN, Cuesta de San José, s/n, 38712 Breña Baja, La Palma, Spain
}

Received 15 June 2017 / Accepted 14 December 2017

\begin{abstract}
Context. Planetary nebulae (PNe) are excellent tracers of stellar populations with low surface brightness, and therefore provide a powerful method to detect and explore the rich system of substructures discovered around the main spiral galaxies of the local group. Aims. We searched the outskirts of the local group spiral galaxy M 33 (the Triangulum) for PNe to gain new insights into the extended stellar substructure on the northern side of the disc and to study the existence of a faint classical halo.

Methods. The search is based on wide field imaging covering a 4.5 square degree area out to a maximum projected distance of about $40 \mathrm{kpc}$ from the centre of the galaxy. The PN candidates are detected by the combination of images obtained in narrowband filters selecting the [OIII] $\lambda 5007 \AA$ and $\mathrm{H} \alpha+[\mathrm{NII}]$ nebular lines and in the continuum $g^{\prime}$ and $r^{\prime}$ broadband filters.

Results. Inside the bright optical disc of M33, eight new PN candidates were identified, three of which were spectroscopically confirmed. No PN candidates were found outside the limits of the disc. Fourteen additional sources showing [OIII] excess were also discovered.

Conclusions. The absence of bright PN candidates in the area outside the galaxy disc covered by this survey sets an upper limit to the luminosity of the underlying population of $\sim 1.6 \times 10^{7} L_{\odot}$, suggesting the lack of a massive classical halo, which is in agreement with the results obtained using the red giant branch population.
\end{abstract}

Key words. planetary nebulae: general - galaxies: individual: M 31 - galaxies: individual: M 33 - local group - galaxies: interactions

\section{Introduction}

Planetary nebulae $(\mathrm{PNe})$ are widely used to trace stellar populations in external galaxies, providing accurate information regarding the luminosity, age, metallicity, and dynamics of their progenitor stellar population. Their bright emission lines, and in particular the [OIII] $\lambda 5007 \AA$ nebular line, provide a distinctive characteristic that makes them easy to identify in continuum-subtracted, narrowband images. Planetary nebulae are especially useful to highlight stellar population in regions of very low surface brightness, such as the outermost regions of galaxies (Corradi et al. 2015) and the intergalactic medium (Longobardi et al. 2015; Arnaboldi et al. 2016) as far as $100 \mathrm{Mpc}$ (e.g. Gerhard et al. 2007).

The rich and complex structures highlighted in recent years in numerous galaxies by extensive broadband imaging surveys have opened a unique laboratory to test not only galactic evolution models but also the history and possible interactions between galaxies in and outside the local group. In this respect, $\mathrm{PNe}$ can play a key role as chemical and dynamical witnesses of these processes.

Assuming a single-star evolutionary scenario, PN progenitors are expected to be low and intermediate mass stars with masses

\footnotetext{
${ }^{\star}$ Based on observations made with the Isaac Newton Telescope and service observations made with the William Herschel Telescope operated on the island of La Palma by the Isaac Newton Group of Telescopes in the Spanish Observatorio del Roque de los Muchachos of the Instituto de Astrofísica de Canarias.
}

between $\sim 0.7$ and $8 M_{\odot}$ (Badenes et al. 2015). During their evolution these stars do not significantly modify abundances of $\alpha$-elements such as oxygen, neon, argon, and sulphur, thereby preserving the original composition of the gas cloud from which the progenitor star formed. Because of their typical progenitor star lifetimes of between 0.1 and $10 \mathrm{Gyr}$, PNe offer a direct look into the past (Magrini et al. 2009). These objects are used to determine metallicity gradients and their temporal evolution by means of comparing the PNe metallicity with those of younger stellar populations, such as HII regions or OB stars (Magrini \& Gonçalves 2009; Annibali et al. 2017).

The kinematics of the stellar population can be inferred by measuring the line-of-sight velocity of a PN population (Arnaboldi 2012 and references therein). Moreover, the [OIII] $\lambda 5007 \AA$ PN luminosity function (PNLF) has been proven to be a robust secondary extragalactic distance candle, providing $10 \%$ accuracy in large galaxies of all Hubble types (Ciardullo 2013).

Deep studies of the bright disc of M 33 confirmed a wealth of HII regions (Wyder et al. 1997; Hodge et al. 1999) and PNe. The first comprehensive search for PNe was performed by Magrini et al. (2000) and provided 131 PN candidates. A subsequent search by Ciardullo et al. (2004) added several new candidates and spectroscopically confirmed $140 \mathrm{PNe}$. The analysis of the spectra pointed out dynamical properties that are consistent with the old disc from the velocity dispersion field of 138 of the PNe; the other $2 \mathrm{PNe}$ are possibly related to a spheroidal component. A detailed discussion of the PNLF of M 33 was included in this 
analysis along with a comparison of the inner and outer disc PNLF, thereby supporting the independence of the bright cutoff with the metallicity suggested in Ciardullo et al. (2002) and displaying a decrease of the PNLF at fainter magnitudes.

The radial metallicity gradient of M33 and its evolution with time was inferred by Magrini et al. (2009) and revisited in Magrini et al. (2016); these works claimed a tight relationship between $\mathrm{O} / \mathrm{H}$ and $\mathrm{Ne} / \mathrm{H}$ abundances and excluded the modification of both elements by the PN progenitors. The comparison between $\mathrm{PNe}$ and HII regions abundances homogeneously analysed in Magrini et al. (2016) indicates a low global enrichment of the M33 disc from the epoch of the formation of the PN progenitors to the present time, following the nearly identical slope of the oxygen gradients of PNe and HII regions.

The PN radial gradient data extend out to $8 \mathrm{kpc}$ from the centre of the galaxy. It would be very interesting to explore whether these measurements can be extended to larger galactocentric distances and to compare these measurements with results obtained with broadband stellar studies of the outskirts of M 33 and test whether a flattening of the gradient is present as found in similar galaxies (e.g. Bresolin et al. 2009). Motivated by the paradigmatic case of M 31, which shows a variety of organized and irregular structures located well beyond its classical disc, and inside which a number of PNe have been found (Merrett et al. 2006), we decided to embark on the search for analogous structures in M 33 using PNe.

Early observations of M33 in the $21 \mathrm{~cm}$ HI line (Rogstad et al. 1976) showed a severe disc warp; this indicates that M33 is not as pristine as it appeared in the optical counterpart known at that time. A first deep optical survey (Ferguson et al. 2007) showed no distortions or substructures on the outskirts of the galaxy, but subsequent and deeper surveys claimed, first, the detection of a stellar halo (Ibata et al. 2007), and then the presence of a large, irregular, low surface brightness stellar substructure surrounding the galaxy (McConnachie et al. 2009, 2010).

McConnachie et al. (2009) found the structure to be composed of an old stellar population $(\langle[\mathrm{Fe} / \mathrm{H}]\rangle \sim-1.6 \mathrm{dex})$, reaching as far as about $40 \mathrm{kpc}$ from the centre in the northwest and southeast quadrants. These authors also suggested that the tidal disruption of M33 in its orbit around M31 would be the cause of the formation of the structure. Further insights about this possible interaction between the two spiral galaxies were gained by means of N-body simulations (McConnachie et al. 2009). The interaction between the two galaxies would have occurred 2-3 Gyr ago and would have triggered a burst of star formation in both galaxies supported by studies of stellar populations located well beyond the limit of their bright classical discs (Barker et al. 2011; Bernard et al. 2012). More recent simulations, however, do not support this scenario (Patel et al. 2017).

The PNe in M33 can be used as an independent tracer of the interaction and star forming episodes, as their brightest representatives are predicted to be produced by stars in an age range covering the period of the supposed M31-M 33 encounter (Schönberner et al. 2007). The presence, or otherwise, of PNe in these regions therefore provides key information about and a quantitative measure of the luminosity of the underlying stellar population (Buzzoni et al. 2006).

\section{Observations}

Images were taken during seven nights on September and October 2013 using the Wide Field Camera (WFC) at the prime focus of the $2.54 \mathrm{~m}$ Isaac Newton Telescope (INT) of the Observatorio del Roque de los Muchachos at the island of La Palma (Spain).

The WFC is an optical mosaic camera consisting of four thinned EEV CCDs with $2048 \times 4096$ pixels each and a scale of 0.33 arcsec pixel $^{-1}$. The size of the mosaic as projected in the sky is 34.2 arcmin with $\sim 1$ arcmin inter-chip gaps.

Seventeen overlapping fields were observed (Fig. 1), covering a total region of 4.5 square degrees and reaching a maximum projected distance of about $40 \mathrm{kpc}$ from the centre of the galaxy.

The emission-line filters used were [OIII], which has a central wavelength and full width at half maximum (FWHM) of 5008 and $100 \AA$, respectively; $\mathrm{H} \alpha+[\mathrm{NII}](6568 / 95 \AA)$; and the broadband $g^{\prime}(4846 / 1285 \AA)$ and $r^{\prime}(6240 / 1347 \AA)$ filters of the Sloan photometric system to evaluate the continuum emission and to distinguish PNe from stars. To allow cosmic ray removal, three exposures were obtained at each telescope position and for each filter, except at one of the telescope positions (M33 17) where only [OIII] and $g^{\prime}$ images could be taken. Exposure times were $1200 \mathrm{~s}$ for both [OIII] and $\mathrm{H} \alpha$, and $400 \mathrm{~s}$ for $r^{\prime}$ and $g^{\prime}$. The seeing during observations ranged from 0.9 to 1.5 arcsec. The observing $\log$ is shown in Table 1.

Spectra of three PNe candidates were obtained in December 2014 in service-queue mode using the auxiliary-port camera and spectrograph ACAM at the $4.2 \mathrm{~m}$ William Herschel Telescope (WHT), also at La Palma. The long slit was opened to $1^{\prime \prime}$ and the exposure time for each object was $\sim 1 \mathrm{~h}$ with the low resolution V400 grism, covering the wavelength range between 3500 and $9400 \AA$ A. The log of these observations is presented in Table 2. Objects presented here are named GCMnn.

\section{Data reduction}

Data were debiased and flat fielded using the ING WFC data reduction pipeline (Irwin \& Lewis 2001). At each telescope position, images were aligned to one of the [OIII] frames, correcting for geometrical distortions. Then all images in the same filter were averaged. These steps were performed using routines available within IRAF ${ }^{1}$. Astrometric solutions were computed using the tasks SCAMP and MISSFITS (Bertin 2006; Marmo \& Bertin 2008) and the SDSS-R9 catalogue (Ahn et al. 2012). The accuracy of astrometry is $\sim 0.1^{\prime \prime}$.

Nights were photometric and instrumental magnitudes for the broadband filters were calibrated by matching stars in the observed fields with the SDSS-R9 catalogue, taking advantage of the large number of stars available. For the narrowband $\mathrm{H} \alpha$ images, we used the same zero point as that derived for the $r^{\prime}$ band, as shown in Drew et al. (2005). The emission-line fluxes in the narrowband filter [OIII] were calibrated using the magnitudes of $31 \mathrm{PNe}$ in Ciardullo et al. (2004), which are included in our fields 16,21 , and 22 . The [OIII] $\lambda 5007 \AA$ magnitudes $\left(m_{5007}\right)$ are related to the monochromatic flux by

$m_{5007}=-2.5 \cdot \log _{10} \cdot F_{5007}-13.74$,

where $F_{5007}$ is expressed in ergs $\mathrm{cm}^{-2} \mathrm{~s}^{-1}$ (Jacoby 1989).

The photometric solution was then extended to all observed fields using the overlapping areas in different pointings. The PNe from Ciardullo et al. (2004) used to calibrate our [OIII]

1 IRAF is distributed by the National Optical Astronomy Observatories, which is operated by the Association of Universities for Research in Astronomy, Inc. (AURA) under cooperative agreement with the National Science Foundations. 


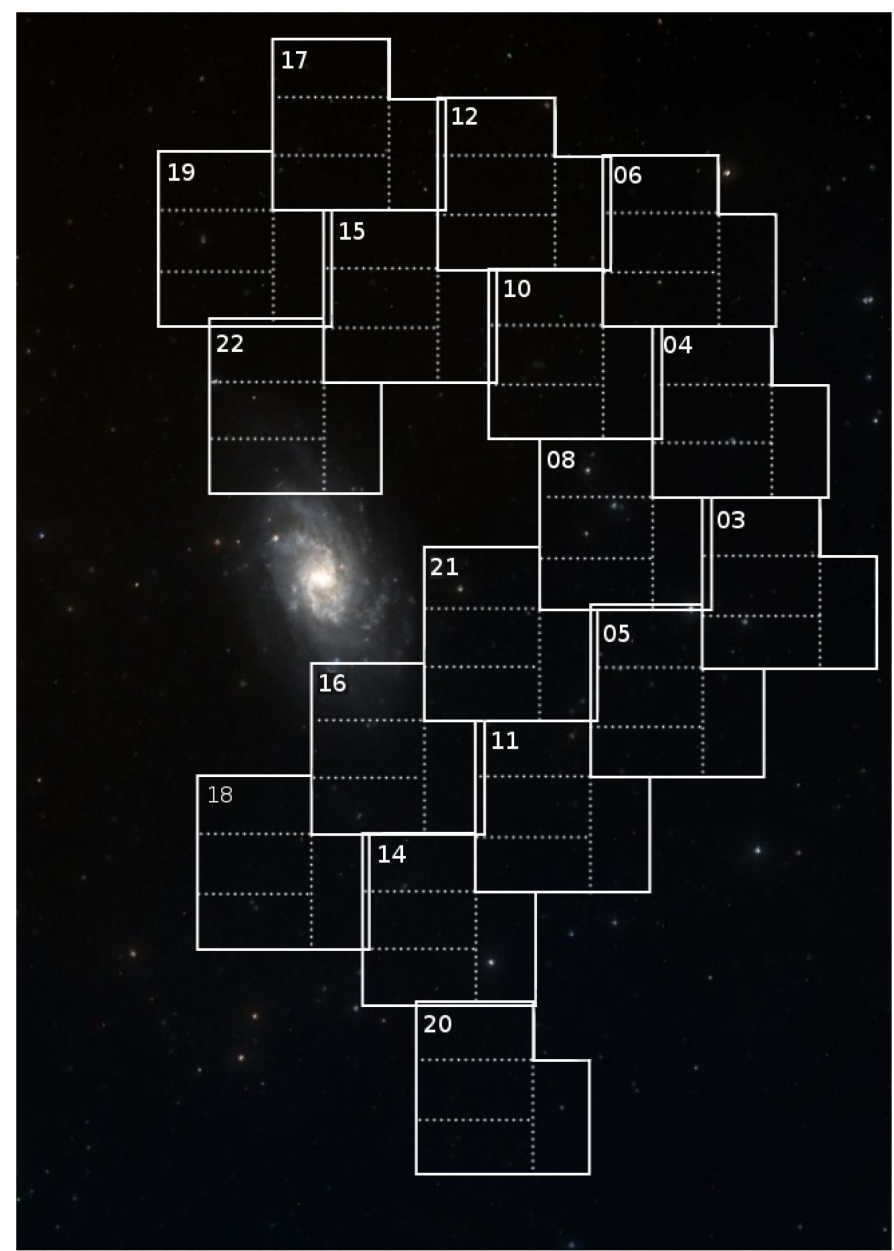

Fig. 1. M33 observed fields. The total field of view of the image is $3^{\circ} \times 4^{\circ}$. North is $u p$, east at left.

Table 1. INT imaging.

\begin{tabular}{cccc}
\hline \hline Field & RA J2000 & Dec J2000 & Date \\
\hline M 33 03 & $01: 27: 16.75$ & $+30: 38: 22.8$ & $2013-10-11$ \\
M33 04 & $01: 27: 59.87$ & $+31: 11: 51.5$ & $2013-10-12$ \\
M 33 05 & $01: 28: 58.07$ & $+30: 17: 46.2$ & $2013-10-11$ \\
M 33 06 & $01: 28: 44.83$ & $+31: 44: 57.9$ & $2013-10-12$ \\
M 33 08 & $01: 29: 43.66$ & $+30: 50: 06.8$ & $2013-09-29$ \\
M33 10 & $01: 30: 29.06$ & $+31: 23: 11.5$ & $2013-09-29$ \\
M33 11 & $01: 30: 41.39$ & $+29: 55: 13.3$ & $2013-10-10$ \\
M33 12 & $01: 31: 15.01$ & $+31: 56: 14.6$ & $2013-10-13$ \\
M33 14 & $01: 32: 23.25$ & $+29: 33: 20.4$ & $2013-10-10$ \\
M33 15 & $01: 32: 58.87$ & $+31: 34: 20.9$ & $2013-09-28$ \\
M33 16 & $01: 33: 09.00$ & $+30: 06: 21.1$ & $2013-10-09$ \\
M33 18 & $01: 34: 50.47$ & $+29: 44: 21.5$ & $2013-10-09$ \\
M33 19 & $01: 35: 29.30$ & $+31: 45: 18.9$ & $2013-09-28$ \\
M33 20 & $01: 31: 34.93$ & $+29: 00: 31.9$ & $2013-10-10$ \\
M33 21 & $01: 31: 27.21$ & $+30: 28: 51.0$ & $2013-10-09$ \\
M33 22 & $01: 34: 42.51$ & $+31: 12: 55.7$ & $2013-09-28$ \\
M33 17 & $01: 33: 45.76$ & $+32: 07: 20.8$ & $2013-10-13$ \\
\hline
\end{tabular}

images and their corresponding identification number and $m_{5007}$ are shown in Table 3. Also shown are the corresponding identification numbers in Magrini et al. (2000) and the final magnitudes obtained for our data after the calibration of the fields.
Table 2. WHT spectroscopy.

\begin{tabular}{ccccc}
\hline \hline Object & RA J2000 & Dec J2000 & $m_{5007}$ & Exp. time (s) \\
\hline GCM 1 & $01: 34: 48.86$ & $+31: 05: 14.8$ & 20.9 & 3400 \\
GCM 2 & $01: 33: 45.20$ & $+30: 21: 22.0$ & 22.7 & 3600 \\
GCM 3 & $01: 33: 52.30$ & $+30: 21: 12.0$ & 23.3 & 3600 \\
\hline
\end{tabular}

Table 3. Known PNe used for the [OIII] calibration.

\begin{tabular}{|c|c|c|c|c|c|}
\hline $\mathrm{ID}^{a}$ & $\mathrm{ID}^{b}$ & RA J2000 & Dec J2000 & $m_{5007^{a}}$ & $m_{5007^{c}}$ \\
\hline 75 & 67 & $01: 33: 52.64$ & $+30: 16: 54.2$ & 21.05 & 21.06 \\
\hline 60 & 59 & $01: 33: 44.38$ & $+30: 20: 23.7$ & 21.15 & 21.22 \\
\hline 66 & 61 & $01: 33: 46.73$ & $+30: 17: 33.5$ & 21.15 & 21.11 \\
\hline 71 & 63 & $01: 33: 50.02$ & $+30: 14: 25.2$ & 21.25 & 21.23 \\
\hline 11 & 13 & $01: 32: 55.04$ & $+30: 09: 52.9$ & 21.34 & 21.34 \\
\hline 152 & - & $01: 35: 13.63$ & $+31: 00: 48.7$ & 21.61 & 21.69 \\
\hline 114 & 89 & $01: 34: 15.72$ & $+31: 08: 12.5$ & 21.72 & 21.79 \\
\hline 117 & 97 & $01: 34: 18.54$ & $+30: 58: 30.5$ & 21.90 & 21.97 \\
\hline 12 & - & $01: 32: 55.11$ & $+30: 14: 01.5$ & 22.23 & 22.28 \\
\hline 134 & 107 & $01: 34: 31.52$ & $+31: 06: 51.3$ & 22.33 & 22.32 \\
\hline 133 & 108 & 01:34:31.49 & $+31: 05: 24.0$ & 22.37 & 22.37 \\
\hline 141 & 121 & $01: 34: 41.96$ & $+30: 56: 49.7$ & 22.41 & 22.39 \\
\hline 142 & 120 & $01: 34: 43.57$ & $+31: 06: 10.7$ & 22.51 & 22.52 \\
\hline 147 & - & $01: 34: 58.18$ & $+31: 06: 47.5$ & 22.51 & 22.27 \\
\hline 148 & - & $01: 35: 04.92$ & $+30: 58: 42.1$ & 22.54 & 22.51 \\
\hline 34 & 33 & $01: 33: 22.85$ & $+30: 13: 41.0$ & 22.59 & 22.49 \\
\hline 6 & 4 & $01: 32: 42.72$ & $+30: 12: 25.5$ & 22.63 & 22.65 \\
\hline 31 & 30 & $01: 33: 21.16$ & $+31: 06: 44.0$ & 22.83 & 22.90 \\
\hline 14 & 15 & $01: 32: 59.40$ & $+30: 10: 24.0$ & & 22.82 \\
\hline 104 & 86 & $01: 34: 11.74$ & $+31: 07: 31.4$ & 22.84 & 22.84 \\
\hline 28 & 27 & $01: 33: 18.48$ & $+30: 12: 38.8$ & 23.23 & 23.25 \\
\hline 15 & 16 & $01: 33: 01.25$ & $+30: 15: 31.1$ & 23.42 & 23.50 \\
\hline 1 & - & 01:32:09.04 & $+30: 22: 05.7$ & 23.51 & 23.49 \\
\hline 100 & 81 & $01: 34: 06.76$ & $+31: 00: 29.3$ & 23.66 & 23.62 \\
\hline 22 & 19 & 01:33:08.99 & $+30: 13: 58.6$ & 23.78 & 23.41 \\
\hline 72 & 64 & $01: 33: 50.82$ & $+30: 18: 43.8$ & 23.90 & 23.90 \\
\hline 80 & 71 & $01: 33: 55.58$ & $+30: 16: 02.9$ & 23.94 & 23.81 \\
\hline 122 & 100 & $01: 34: 22.96$ & $+30: 59: 32.9$ & 24.07 & 24.16 \\
\hline 129 & 102 & $01: 34: 25.83$ & $+31: 07: 44.5$ & 24.28 & 24.45 \\
\hline 58 & 57 & $01: 33: 43.48$ & $+30: 59: 41.1$ & 24.42 & 24.18 \\
\hline 25 & 25 & $01: 33: 15.50$ & $+30: 17: 53.7$ & 24.51 & 24.42 \\
\hline
\end{tabular}

References. ${ }^{(a)}$ Ciardullo et al. (2004); ${ }^{(b)}$ Magrini et al. (2000); ${ }^{(c)}$ this work.

Photometric errors derived internally from DAOPHOT, photon statistics, and background uncertainties are small and generally lower than the typical systematic errors. We have to account for the uncertainty from the calibration sources in Ciardullo et al. (2004) and the dispersion term for each calculated zero point, including the systematic error of their propagation. Adding all of the sources of uncertainty through least squares, an error up to $\sim 0.1 \mathrm{mag}$ for $m[\mathrm{OIII}] \leq 24.5$ was estimated, increasing to 0.4 mag for $m[\mathrm{OIII}] \leq 25.5$. These errors are larger for faintest sources.

Spectra were reduced (debiased, overscan corrected, trimmed, flat fielded, combined, and finally sky subtracted and extracted) using the packages CCDRED and TWODSPEC in IRAF. Before subtracting the sky background, cosmic rays were eliminated using the task lacos spec (van Dokkum 2001). The 
Table 4. PNe candidates.

\begin{tabular}{ccccccc}
\hline \hline Object & RA J2000 & Dec J2000 & $d(\mathrm{kpc})$ & $m_{5007}$ & $\mathrm{~F}([\mathrm{OIII}]) / \mathrm{F}(\mathrm{H} \alpha)$ & Comments \\
\hline GCM 1 & $01: 34: 48.84$ & $+31: 05: 14.9$ & 6.99 & 20.9 & 2.39 & Confirmed PN*, WHT spectra \\
GCM 2 & $01: 33: 45.18$ & $+30: 21: 21.4$ & 4.83 & 22.7 & 0.73 & Confirmed PN, WHT spectra \\
GCM 3 & $01: 33: 52.30$ & $+30: 21: 12.2$ & 5.06 & 23.3 & 1.57 & Confirmed PN, WHT spectra \\
GCM 4 & $01: 33: 11.37$ & $+30: 21: 12.6$ & 4.96 & 24.0 & 0.52 & Crowded region \\
GCM 5 & $01: 32: 17.29$ & $+30: 41: 35.0$ & 8.00 & 25.2 & 1.80 & Visual search. Extremely faint. \\
GCM 6 & $01: 33: 11.52$ & $+31: 05: 49.2$ & 8.83 & 25.4 & 0.72 & Visual search. Extremely faint. \\
GCM 7 & $01: 34: 18.25$ & $+30: 58: 27.2$ & 4.85 & 25.7 & 0.63 & Visual search. Extremely faint. \\
GCM 8 & $01: 33: 11.22$ & $+30: 18: 28.8$ & 5.57 & 24.4 & - & Visual search. Crowded region \\
\hline
\end{tabular}

Notes. ${ }^{(*)}$ PN128, classified as "possible PN" in Magrini et al. (2000).

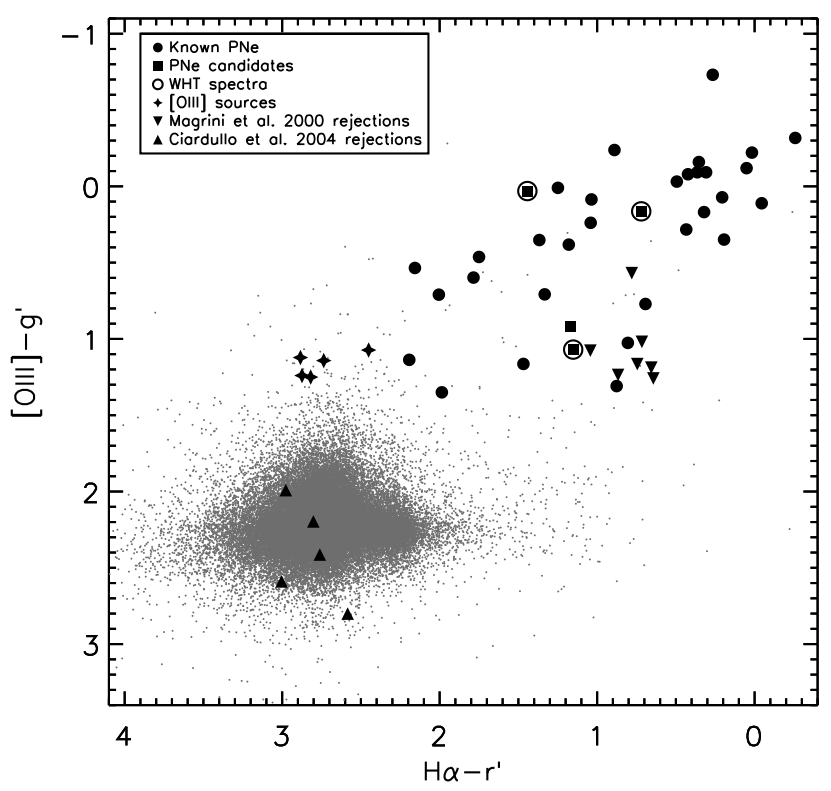

Fig. 2. Colour-colour diagram of the sources in the sampled area of M33.

Table 5. PNe misidentified in Ciardullo et al. (2004).

\begin{tabular}{lcc}
\hline \hline Object & RA J2000 & Dec J2000 \\
\hline PN 151 & $01: 35: 10.50$ & $+31: 05: 14.9$ \\
PN 144 & $01: 34: 45.50$ & $+31: 01: 11.7$ \\
PN 120 & $01: 34: 20.50$ & $+31: 10: 52.0$ \\
PN 97 & $01: 34: 05.54$ & $+31: 12: 30.1$ \\
PN 61 & $01: 33: 44.62$ & $+31: 04: 03.5$ \\
PN 42 & $01: 33: 27.89$ & $+31: 06: 22.3$ \\
\hline
\end{tabular}

flux calibration was carried out using the standard star Feige 15 (Stone 1977). More details are given in Sect. 5.2.

\section{Data analysis}

The PN search was made in two different ways: by visual inspection of the combined set of narrow and broadband images and via the analysis of colour-colour diagrams from automated photometric measurements.

As mentioned before, 31 of the known PNe in M33 are located in a region of about 0.4 square degrees close to the bright disc of the galaxy, which is a zone that was partially covered by three of our mapped regions. Those PNe were used as a reference sample for the visual and photometric searches.

\subsection{Visual search}

The $g^{\prime}$ images were used as the reference continuum for the [OIII] frames and the $r^{\prime}$ images were used as reference continuum for $\mathrm{H} \alpha$. Various display colours were assigned to images in different filters to create colour-composite images in which we searched for sources with $\mathrm{H} \alpha$ and [OIII] excesses. Specifically, red was assigned to the wide filter $g^{\prime}$ or $r^{\prime}$, and green to the narrow filter [OIII] or $\mathrm{H} \alpha$, which makes point-source emission-line objects appear as outstanding green dots in both the $[\mathrm{OIII}] / g^{\prime}$ and $\mathrm{H} \alpha / r^{\prime}$ colour-composite images, whereas normal stars appear with a nearly homogeneous orange colour.

In this way, we identified $8 \mathrm{PN}$ candidates, showing both [OIII] and $\mathrm{H} \alpha$ excesses, and 2 additional objects, showing only an [OIII] excess. All these candidates are located at distances smaller than $\sim 9 \mathrm{kpc}$ from the centre of M 33, i.e. inside the bright optical disc of the galaxy. At distances larger than $9 \mathrm{kpc}$, no objects with both an [OIII] and $\mathrm{H} \alpha$ emission were found. However, 14 additional sources were detected in those external areas with just an [OIII] excess and lower or negligible emission in the other three filters.

\subsection{Colour-colour diagram}

Sources in each of the [OIII] images were automatically searched using SExtractor (Bertin \& Arnouts 1996), setting the detection threshold to the limit of $2 \sigma$ over the background. This ensures that very faint sources are recovered, but includes a large number of artefacts. Automatic aperture photometry was then performed via DAOPHOT (Stetson 1987) with an empirically determined optimum aperture of $\sim 5 / 2$ times the stellar FWHMs.

Photometry of $\sim 100000$ stars was obtained and the results were analysed using $\mathrm{H} \alpha-r^{\prime}$ versus [OIII] $g^{\prime}$ colour-colour diagrams (Fig. 2), which are particularly suited for selecting PNe (see e.g. Corradi et al. 2005).

In this kind of diagram, PNe show up as objects with strong [OIII] and $\mathrm{H} \alpha$ excess compared to the bulk of normal stars, as indicated by the filled circles in Fig. 2, which show the location in the colour-colour diagram of the photometry of the known $\mathrm{PNe}$ in the observed fields. All detected sources in this area were visually inspected in the individual images to reject artefacts or poor-photometry sources. This check was also extended to sources with just [OIII] excess. In this way, 4 new PNe candidates were identified, all of which were independently found by our visual search. In addition, 5 of the 14 sources mentioned 
Table 6. Rejected objects classified as "possible PNe" by Magrini et al. (2000).

\begin{tabular}{lccl}
\hline \hline Object & RA J2000 & Dec J2000 & Comments \\
\hline PN23 & $01: 33: 13.54$ & $+30: 22: 36.1$ & Extended, IR counterpart (w3, w4) \\
PN5 & $01: 32: 44.16$ & $+30: 22: 03.9$ & IR counterpart (w3, w4) \\
PN127 & $01: 34: 47.10$ & $+30: 59: 36.2$ & Extended, Galaxy 2247 \\
PN78 & $01: 34: 02.55$ & $+30: 58: 10.5$ & Extended, IR counterpart (w3, w4), CO counterpart \\
PN55 & $01: 33: 41.77$ & $+30: 08: 31.3$ & Extended, IR counterpart (w1, w2) \\
Em24 & $01: 33: 10.78$ & $+30: 18: 08.8$ & Globular cluster \\
Em59 & $01: 34: 42.52$ & $+30: 55: 44.4$ & Extended, globular cluster candidate \\
\hline
\end{tabular}

Table 7. Sources showing a substantial [OIII] excess.

\begin{tabular}{lccccl}
\hline \hline Object & RA J2000 & Dec J2000 & $d(\mathrm{kpc})$ & $m_{5007}$ & Comments \\
\hline GCM E1 & $01: 33: 02.82$ & $+30: 03: 15.5$ & 9.32 & 24.8 & Detected in $g^{\prime}$ and $r^{\prime}$. No H $\alpha$. Visual search. \\
GCM E2 & $01: 27: 35.41$ & $+31: 00: 08.2$ & 33.60 & 24.1 & Detected in $g^{\prime}$ and $r^{\prime}$. No H $\alpha$. Visual search. \\
GCM E3 & $01: 31: 27.30$ & $+31: 47: 39.5$ & 25.24 & 24.8 & Detected in $g^{\prime}$. No H $\alpha$, no $r^{\prime}$. Visual search. \\
GCM E4 & $01: 28: 26.44$ & $+31: 39: 31.8$ & 35.93 & 24.7 & No H $\alpha$, no $r^{\prime}$, no $g^{\prime}$. Visual search. \\
GCM E5 & $01: 29: 48.03$ & $+30: 55: 06.1$ & 22.03 & 24.7 & Detected in $g^{\prime}$ and $r^{\prime}$. No H $\alpha$. Visual search. \\
GCM E6 & $01: 29: 54.43$ & $+30: 55: 17.2$ & 21.54 & 25.4 & Detected in $g^{\prime}$ and $r^{\prime}$. No H $\alpha$. Visual search. \\
GCM E7 & $01: 34: 09.30$ & $+29: 37: 10.3$ & 17.63 & 24.9 & Detected in $g^{\prime}$ and $r^{\prime}$. No H $\alpha$. Visual search. \\
GCM E8 & $01: 30: 31.82$ & $+31: 26: 45.3$ & 24.17 & 24.8 & Detected in $g^{\prime}$. No H $\alpha$, no $r^{\prime}$. Visual search. \\
GCM E9 & $01: 33: 14.80$ & $+30: 10: 39.4$ & 7.42 & 25.4 & Bright in $r^{\prime}, g^{\prime}, \mathrm{H} \alpha$. Visual search. \\
GCM E10 & $01: 33: 25.78$ & $+31: 04: 51.6$ & 7.86 & 24.0 & Bright in $r^{\prime}, g^{\prime}, \mathrm{H} \alpha$. \\
GCM E11* & $01: 32: 38.31$ & $+29: 26: 02.5$ & 18.85 & 24.6 & Bright in $r^{\prime}, g^{\prime}, \mathrm{H} \alpha$. \\
GCM E12 & $01: 31: 55.81$ & $+31: 51: 51.8$ & 24.66 & 24.3 & Bright $r^{\prime}, g^{\prime}, \mathrm{H} \alpha$. \\
GCM E13 & $01: 30: 32.15$ & $+31: 26: 18.7$ & 24.05 & 24.9 & Bright $r^{\prime}, g^{\prime}, \mathrm{H} \alpha$. \\
GCM E14 & $01: 30: 24.77$ & $+29: 39: 18.6$ & 19.32 & 23.8 & Bright $r^{\prime}, g^{\prime}, \mathrm{H} \alpha$. \\
\hline
\end{tabular}

Notes. ${ }^{(*)}$ J013238.33+292602.57, catalogued as quasar at $z \sim 4.45$ by Wang et al. (2016). It should be revised with the detected [OIII] excess.

above, showing just [OIII] excess, were also recovered by the photometry study within relatively low errors.

Some candidates found by visual inspection are not recovered by the photometric search. The main reason is that they are exceedingly faint in the broadband filters. In some other cases, they are lost because of confusion in very crowded areas.

\subsection{Contaminants}

The degree of contamination by spurious sources is especially high in the crowded regions of the galaxy and close to spiral arms. This is mostly caused by the presence of giant HII regions and the associated high stellar background. Because of the low detection threshold adopted, spurious PN candidates were also found in less crowded regions, mainly at the faintest magnitudes, owing to large photometric errors. Detector cosmetics, cosmic rays, and transient effects such as asteroids or satellite tracks also contribute to contamination.

In order to clean the colour-colour diagram from contaminants, we used the following additional information. At the distance of M33 (840 kpc; Freedman et al. 2001), we do not expect to resolve any PN (Magrini et al. 2000). Therefore every extended source is not considered a PN. Besides, even though PNe on the Milky Way show a significant emission in the IR, it is not expected that PNe at the distance of M 33 would be detected by standard all-sky surveys. Every source with an IR counterpart in the WISE archive is therefore rejected as a PN. Symbiotic stars can mimic PNe in this survey. Indeed, Mikołajewska et al. (2017) discovered 12 symbiotic stars in M 33.
Only one of these stars, M33 SySt-11, is associated with a faint WISE source, which is only detected in the shorter w1 and w2 bands. The sources showing IR emission (Table 6) are therefore unlikely to be symbiotic stars.

Even after applying these criteria, some dubious sources remain, and we have visually inspected and searched in catalogues and the literature, all candidates that fall in the selected regions of the colour-colour diagram.

\section{Results}

\subsection{New and rejected PN candidates}

Eight PN candidates were identified by combining the photometric and visual search. Their coordinates, deprojected distances from the centre of the galaxy, and [OIII] $\lambda 5007 \AA$-to-H $\alpha+[\mathrm{NII}]$ line flux ratios $R$ are shown in Table 4. To compute the deprojected galactocentric distances, the galaxy distance of $840 \mathrm{kpc}$ (Freedman et al. 2001), an intermediate inclination of $53^{\circ}$, and the position angle of $22^{\circ}$ are assumed (Makarov et al. 2014). All candidates have an $R$ ratio between the limits $0.3<R<3$ established by Ciardullo et al. (2002) for PNe within 3 magnitudes from the cut-off of the PNLF. We note that the value of this cut to exclude HII regions decreases at fainter magnitudes (Merrett et al. 2006).

It is important to emphasize that every previously known PN located in our fields were recovered by our search. However, based on our measured colours, we did not confirm some of the candidates in Ciardullo et al. (2004). These are listed in 
Table 8. Observed and dereddened fluxes.

\begin{tabular}{|c|c|c|c|c|c|}
\hline Object & $\mathrm{c}$ & Ion & $\lambda_{\text {rest }}(\AA)$ & $F_{\lambda}$ & $I_{\lambda}$ \\
\hline \multirow[t]{13}{*}{ GCM 1} & $0.08 \pm 0.02$ & $\mathrm{HI}(\mathrm{H} \beta)$ & 4861.3 & $100 \pm 6$ & $100 \pm 6$ \\
\hline & & [OIII] & 4958.9 & $330 \pm 17$ & $328 \pm 17$ \\
\hline & & [OIII] & 5006.8 & $1000 \pm 50$ & $992 \pm 50$ \\
\hline & & $\mathrm{HeI}$ & 5875.6 & $17 \pm 2$ & $16 \pm 2$ \\
\hline & & [NII] & 6548.0 & $13 \pm 2$ & $12 \pm 2$ \\
\hline & & $\mathrm{HI}(\mathrm{H} \alpha)$ & 6562.8 & $302 \pm 16$ & $286 \pm 16$ \\
\hline & & [NII] & 6583.4 & $28 \pm 2$ & $26 \pm 2$ \\
\hline & & $\mathrm{HeI}$ & 6678.2 & $4.2 \pm 1.2$ & $4.0 \pm 1.1$ \\
\hline & & [SII] & $6717+6731$ & $3.8 \pm 1.4$ & $3.6 \pm 1.3$ \\
\hline & & HeI & 7065.3 & $11 \pm 2$ & $10 \pm 2$ \\
\hline & & [ArIII] & 7135.8 & $12 \pm 1$ & $11 \pm 1$ \\
\hline & & [OII] & 7323.0 & $13 \pm 2$ & $12 \pm 2$ \\
\hline & & [ArIII] & 7751.1 & $3.4 \pm 1.4$ & $3.2 \pm 1.3$ \\
\hline \multirow[t]{13}{*}{ GCM 2} & $0.17 \pm 0.10$ & $\mathrm{HI}(\mathrm{H} \beta)$ & 4861.3 & $100 \pm 12$ & $100 \pm 12$ \\
\hline & & [OIII] & 4958.9 & $581 \pm 40$ & $575 \pm 35$ \\
\hline & & [OIII] & 5006.8 & $1613 \pm 90$ & $1589 \pm 90$ \\
\hline & & {$[\mathrm{NI}]$} & 5200.3 & $84 \pm 11$ & $81 \pm 10$ \\
\hline & & [NII] & 5755.0 & $41 \pm 14$ & $38 \pm 13$ \\
\hline & & $\mathrm{HeI}$ & 5875.6 & $27 \pm 8$ & $25 \pm 8$ \\
\hline & & {$[\mathrm{OI}]$} & 6300.3 & $102 \pm 13$ & $93 \pm 13$ \\
\hline & & [NII] & 6548.0 & $496 \pm 40$ & $442 \pm 40$ \\
\hline & & $\mathrm{HI}(\mathrm{H} \alpha)$ & 6562.8 & $321 \pm 30$ & $286 \pm 30$ \\
\hline & & [NII] & 6583.4 & $1342 \pm 70$ & $1194 \pm 100$ \\
\hline & & [SII] & $6717+6731$ & $130 \pm 16$ & $115 \pm 15$ \\
\hline & & [ArIII] & 7135.8 & $31 \pm 9$ & $26 \pm 8$ \\
\hline & & [OII] & 7323.0 & $37 \pm 11$ & $32 \pm 10$ \\
\hline \multirow[t]{6}{*}{ GMC3 } & $0.00 \pm 0.24$ & $\mathrm{HI}(\mathrm{H} \beta)$ & 4861.3 & $100 \pm 21$ & $100 \pm 21$ \\
\hline & & [OIII] & 4958.9 & $363 \pm 30$ & $363 \pm 30$ \\
\hline & & [OIII] & 5006.8 & $992 \pm 60$ & $992 \pm 60$ \\
\hline & & [NII] & 6548.0 & $51 \pm 11$ & $51 \pm 11$ \\
\hline & & $\mathrm{HI}(\mathrm{H} \alpha)$ & 6562.8 & $265 \pm 23$ & $265 \pm 23$ \\
\hline & & [NII] & 6583.4 & $145 \pm 16$ & $145 \pm 16$ \\
\hline
\end{tabular}

Table 5. Five of these six rejections are shown in the colourcolour diagram of Fig. 2 as filled upward triangles. The sixth unconfirmed candidate shows big photometric errors and is rejected as a possible PN after visual inspection. For the 31 known PNe adopted as the reference sample, the $\mathrm{H} \alpha+[\mathrm{NII}]$ measured fluxes (not shown here) are brighter than in the Ciardullo et al. (2004) results. As explained in Ciardullo et al. (2004), when compared with Magrini et al. (2000), this is partly due to the differing contributions of [NII] within the bandpasses of the filters.

Several candidates with apparently good colour data were also rejected after visual inspection. Seven of these candidates are objects previously found by Magrini et al. (2000; filled downward triangles in Fig. 2): five were listed as "possible PNe" by Magrini et al. (2000), which had been previously discarded as PNe by Ciardullo et al. (2004), and two more were catalogued as emission-line objects with non-negligible continuum (Table 6). Additional compact sources with only [OIII] excess are listed in Table 7. Some have an $\mathrm{H} \alpha$ and $r^{\prime}$ counterpart, but show no detectable $\mathrm{H} \alpha$ excess. These could be compact galaxies with [OII] $\lambda 3727 \AA$ emission redshifted to $z \sim 0.35$ or Ly $\alpha$ emitters at $z \sim 3.1$. Another less likely possibility is that these sources are M33 H-deficient PNe. Further studies of these sources are of interest. The locations of the discovered objects and the previously known PNe are shown in Fig. 3. Finding charts for our PNe candidates (Table 4) are shown in Fig. 5, whereas images of three [OIII] excess sources (Table 7) are shown in Fig. 6. Finally, Fig. 7 shows images at the four bands of a PN and an excess [OIII] source.

\subsection{Spectroscopic confirmation of $P N$ candidates}

The three brightest candidates were spectroscopically confirmed using ACAM at the WHT. Spectra are shown in Fig. 4. Relative flux calibration was carried out using the standard star Feige 15 (Stone 1977), observed on the night of December 27, 2014.

Emission-line fluxes were measured using the package SPLOT of IRAF. Errors in the fluxes were estimated adding the measured statistical errors quadratically to a constant $5 \%$ term taken as representative of systematic errors in the flux calibration. Observed line fluxes were corrected for the effect of the interstellar extinction using the extinction law of Cardelli et al. (1989) with $R_{v}=3.1$. The logarithmic nebular extinction $\mathrm{c}(\mathrm{H} \beta)$ was derived with the observed-to-theoretical Balmer ratio $\mathrm{H} \alpha / \mathrm{H}(\beta)$ from Osterbrock \& Ferland (2006).

Figure 4 shows that all bright emission lines typical of $\mathrm{PNe}$ are detected in our low resolution spectra that have [OIII] much brighter than $\mathrm{H} \alpha$. Also, in the $\log (\mathrm{H} \alpha /[\mathrm{SII}] \lambda \lambda 6717+6731)$ 


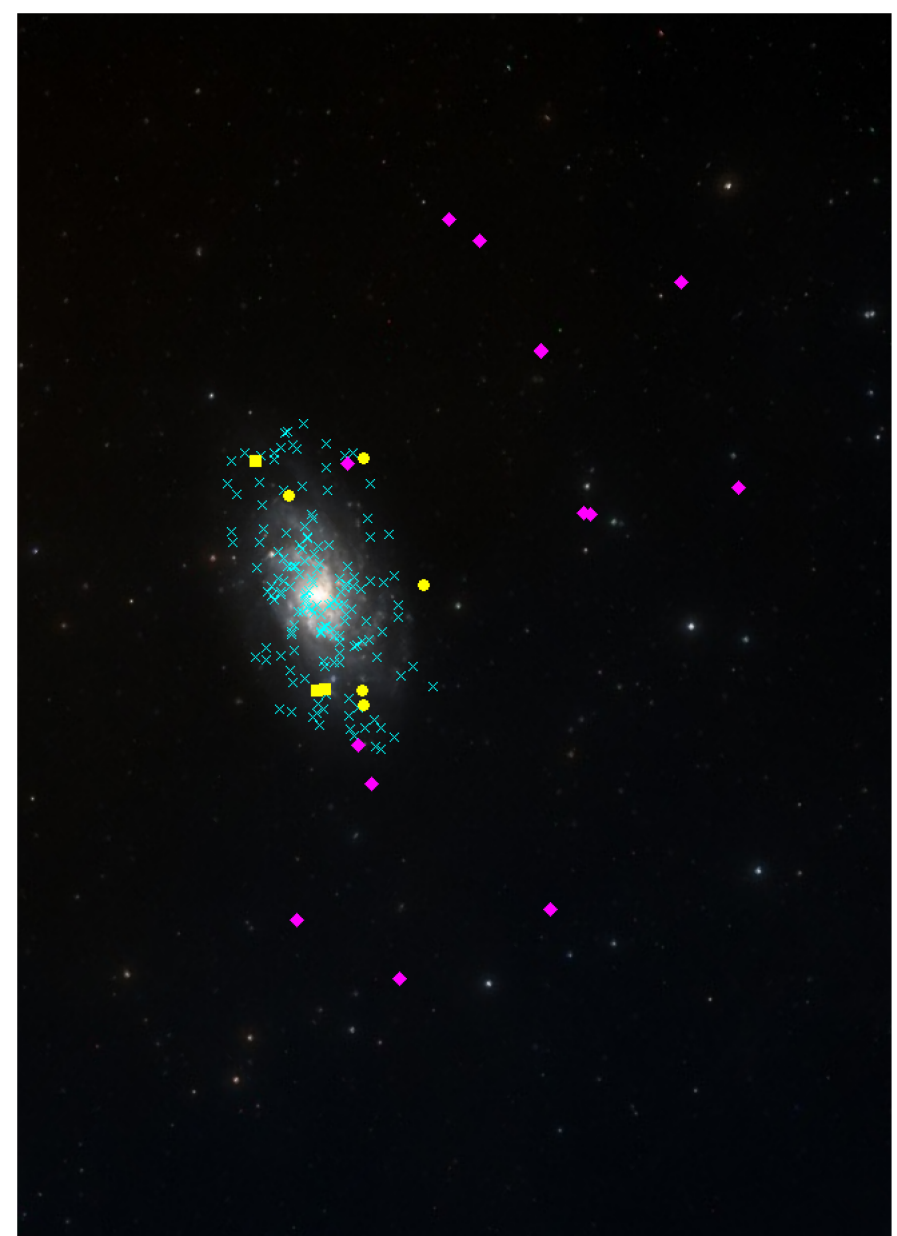

Fig. 3. Location of the discovered objects. The yellow filled circles indicate PNe candidates. The yellow filled squares indicate the confirmed $\mathrm{PNe}$ and magenta rhombi are candidates with an [OIII] excess. Previous known PNe are shown with cyan small crosses. The total field of view of the image is $3^{\circ} \times 4^{\circ}$. North is up, east at left.

versus $\log (\mathrm{H} \alpha /[\mathrm{NII}] \lambda 6583)$ diagnostic diagram (Viironen et al. 2007) GCM 1 and 2 can be located near to the region populated by PNe. We note that GCM 1 was previously identified by Magrini et al. (2000) as a possible PN candidate, but rejected by Ciardullo et al. (2004) because of its bright emission in their wide filters; in addition, GCM 2 shows a remarkably high [NII] $\lambda 6548$ and $\lambda 6584$ flux relative to $\mathrm{H} \alpha$, suggesting it is a type I PN.

Given the faintness of the PNe detected lines and limited spectral resolution, no reliable determination of electron temperature $\left(T_{\mathrm{e}}\right)$ and electron density $\left(N_{\mathrm{e}}\right)$ is possible.

\subsection{Completeness limit}

The completeness limit of our search was estimated by adding artificial stars with various magnitudes in both narrowband and continuum images and then computing the recovery rate of such artificial objects (Minniti \& Zijlstra 1997; Magrini et al. 2002, 2003; Corradi et al. 2005). A recovery rate of $50 \%$ defines incompleteness.

For [OIII] and $\mathrm{H} \alpha$, the completeness limit in our observed regions is between 25.5 and $26.0 \mathrm{mag}$. In the $\sim 0.4$ square degree area closer to the bright disc of the galaxy, this completeness limit typically increases by 0.5 magnitudes, owing to the higher background and crowdedness. For $g^{\prime}$ and $r^{\prime}$, the completeness limit is at $24.5 \mathrm{mag}$, becoming almost one magnitude brighter, $\sim 23.5$, in that 0.4 square degree area closer to the bright disc of M33.

\subsection{PN population size and luminosity of the external regions of $M 33$}

The luminosity of a stellar population that produces $\mathrm{PNe}$ can be determined using the $\alpha$ ratio, i.e. the luminosity-specific PN number density (Jacoby 1980). This quantity links the PNe population size with the bolometric luminosity of the parent stellar population. Buzzoni et al. (2006) discusses the method in detail and provides recipes to determine $\alpha$ as a function of the age and metallicity (colour) of the host galaxy.

Based on the simple stellar population models by Renzini \& Buzzoni (1986), the number of PNe related to its progenitor stellar population can be expressed in terms of the PN visibility lifetimes $\left(\tau_{\mathrm{PN}}\right)$ as follows:

$\alpha=\frac{N_{\mathrm{PN}}}{L_{\mathrm{TOT}}}=B \cdot \tau_{\mathrm{PN}}$,

where $B$ is the specific evolutionary flux (stars $\mathrm{yr}^{-1} L_{\odot}^{-1}$ ), that is only weakly dependent on the metallicity (except at very low metallicities).

Given that no PN candidates are found outside the bright M33 disc down to the completeness limit of our survey, we can estimate an upper limit to the total stellar luminosity at the outer regions that we have imaged. For that, we adopt a standard shape for the PNLF and a completeness limit of $25.5 \mathrm{mag}$, which corresponds to 5 magnitudes below the bright cut-off of the PNLF assuming an absolute magnitude cut-off of $M^{*}=-4.47$ (Ciardullo et al. 2002) at the distance of M 33 (Ciardullo et al. 2004). Also, we assume that the faintest detectable PNe are about 8 magnitudes below the bright cut-off $m *$ of the PNLF (Buzzoni et al. 2006).

From previous deep studies of M 33 we know that the colour of the external regions is $(B-V)_{\text {outskirts }} \sim 0.55$, corresponding to $\log \alpha \approx-6.8$ (Buzzoni et al. 2006). With all these figures, we infer an upper limit for the luminosity of the observed 4.5 square degree region of $\sim 1.6 \times 10^{7} L_{\odot}$.

This agrees with a recent deep study of the red giant branch (RGB) population in M33, setting a limit to the luminosity of a possible halo to $<1.6 \times 10^{7} L_{\odot}$ (McMonigal et al. 2016) but leaving open the alternative existence of a very extended and faint thick disc (Barker et al. 2011; Cockcroft et al. 2013). This value is smaller than, but roughly compatible with, the luminosity of the 40 square degrees substructure surrounding M33 $\left(\sim 1.9 \times 10^{7} L_{\odot}\right)$ measured by McConnachie et al. (2009) based also on the study of their RGB population. In the past, several works have claimed the detection of a halo in M33. Studies of RR Lyrae stars suggested the existence of this halo (Sarajedini et al. 2006; Yang et al. 2010); subsequent works using metallicity indicators have also suggested its existence (Pritzl et al. 2011). Globular clusters (Chandar et al. 2002) and AGB and RGB population studies (e.g Barker et al. 2007a,b; Ibata et al. 2007 or Cioni et al. 2008) also claimed tentative detections of the halo. However, the present work and that from McMonigal et al. (2016) have failed to measure any bright stellar component in the external regions of the galaxy, excluding the existence of a massive classical monolithic halo. 


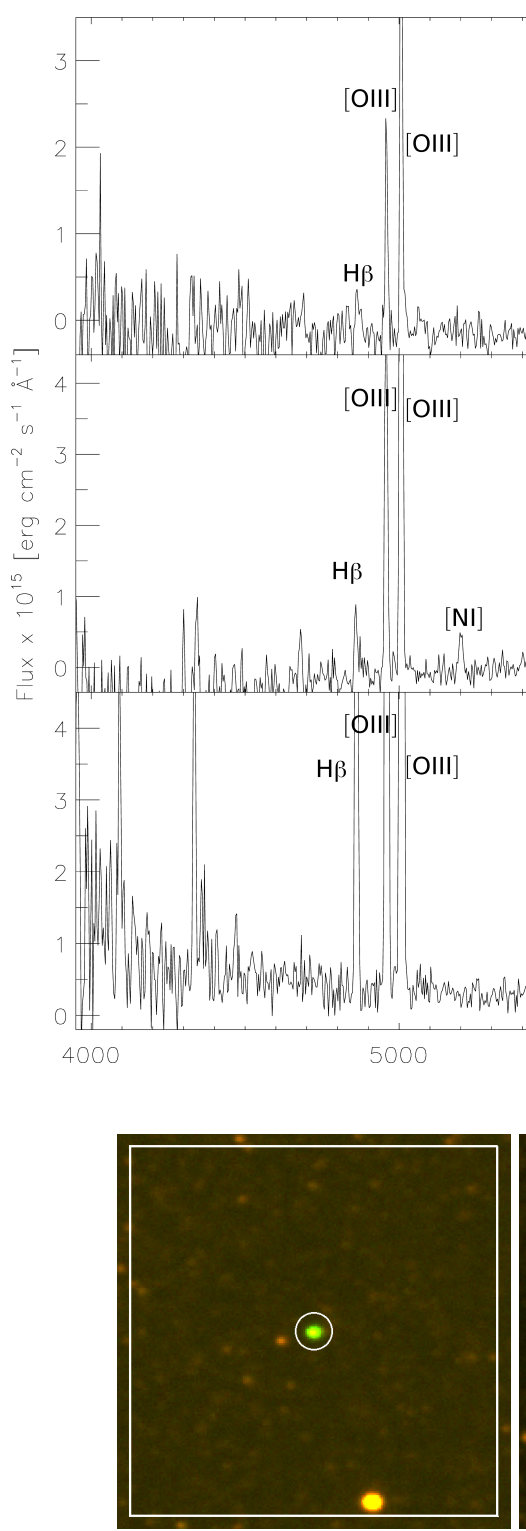

(a) GCM1

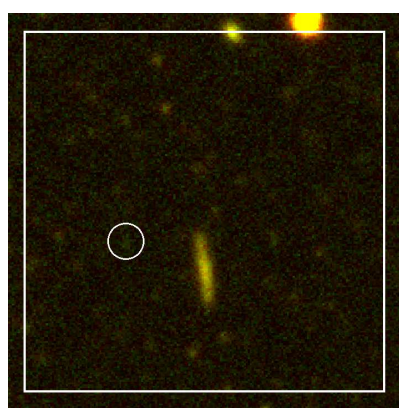

(e) GCM5

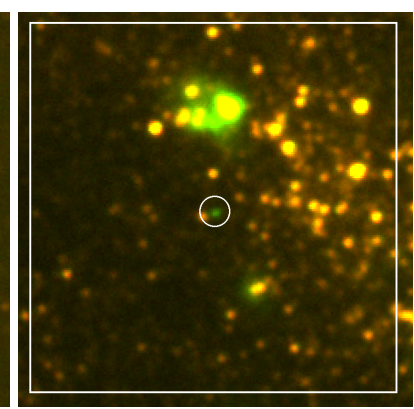

(b) GCM2

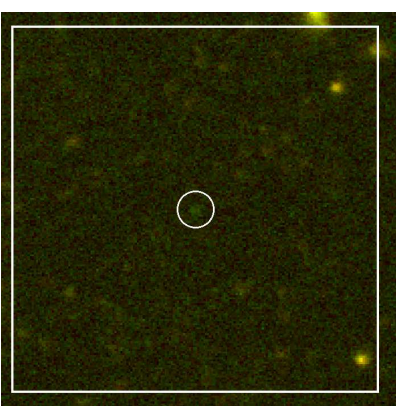

(f) GCM6

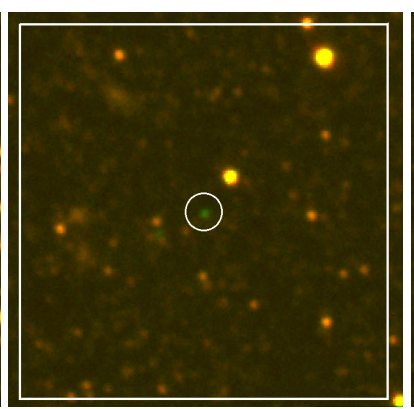

(c) GCM3

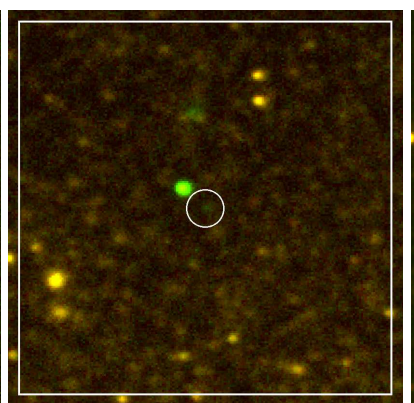

(g) GCM7
Fig. 4. WHT spectra of three new PNe. We note the extremely bright [NII] lines in GCM2.

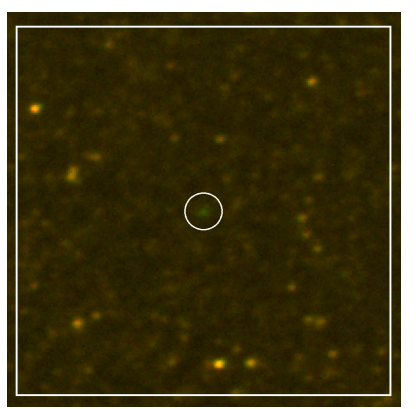

(d) GCM4

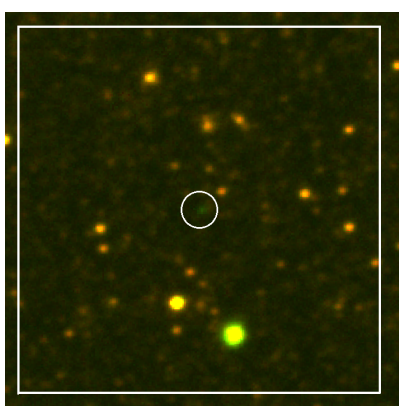

(h) GCM8

Fig. 5. Finding charts for the PNe candidates (green: [OIII], red: $g^{\prime}$ ). North is $u p$, east at left. Field size is $1^{\prime} \times 1^{\prime}$.

\section{Summary and conclusions}

Planetary nebulae are known to be excellent tracers of stellar populations even in very low surface brightness regions. Taking advantage of this property, we have carried out the first deep search for PNe in the outer regions of M33 to gain further insights into the existence of a classical halo or, as in the case of M31, of a rich system of extended substructures possibly related to past interactions between the two galaxies.

The lack of reliable PN candidates in the observed external regions of $\mathrm{M} 33$, and down to the completeness limit of our search of $25.5 \mathrm{mag}$ in [OIII] and $\mathrm{H} \alpha$, poses an upper limit to the luminosity of any stellar population in this area 


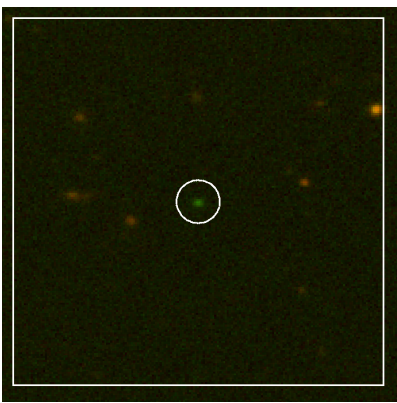

(a) GCM E2

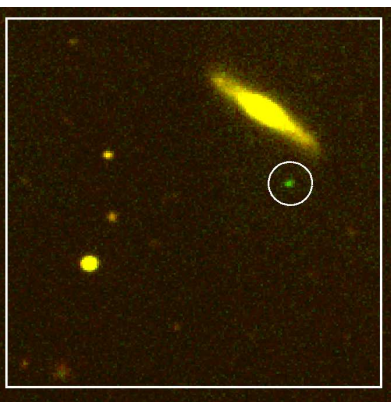

(b) GCM E5

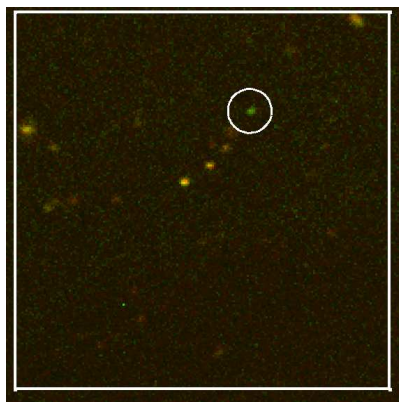

(c) GCM E6

Fig. 6. As in Fig. 5 for three sources with [OIII] excess.

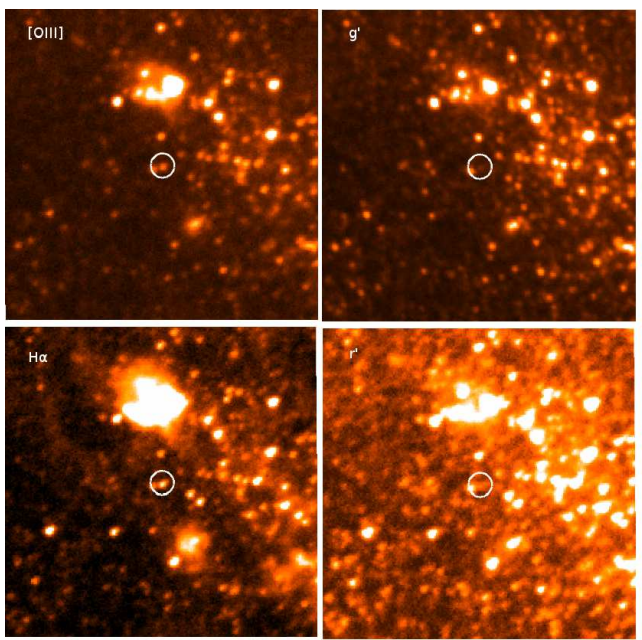

(a) GCM2 (PN).

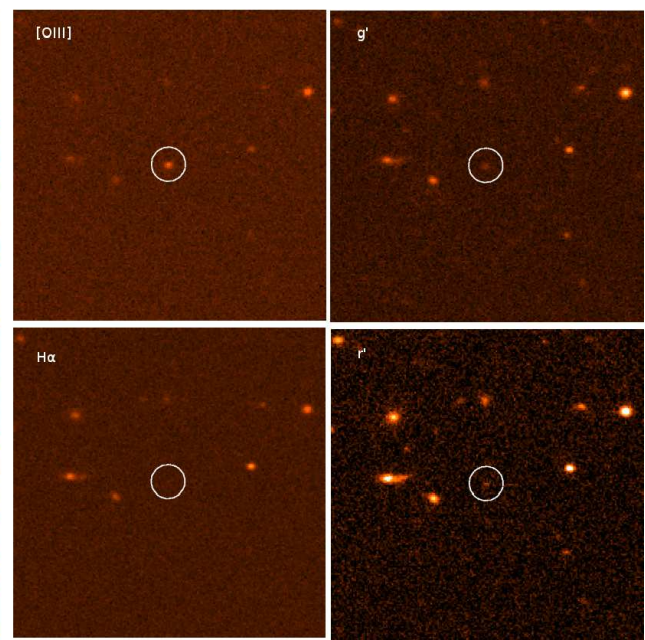

(b) GCM E2 ([OIII] excess source).

Fig. 7. Two representative sources imaged at the four filters. North is $u p$, east at the left. Field size is $1^{\prime} \times 1^{\prime}$.

of $L_{\text {outskirts, } \mathrm{PNe}}<1.6 \times 10^{7} L_{\odot}\left(M_{\mathrm{V}} \sim-12.45\right)$. It can therefore be concluded that there is no evidence of prominent extended structures outside its bright disc, and particularly, of a massive classical halo, in line with results obtained using other stellar tracers.

The difference with M 31, which instead displays a very rich circumgalactic environment populated by bright $\mathrm{PNe}$, is therefore substantial, and goes against the idea of a strong tidal interaction in a past encounter of the two galaxies (Patel et al. 2017).

Acknowledgements. We deeply appreciate discussion with Bruce Balick and Karen Kwitter, which led to the development of this work. We thank Robert Greimel for the first treatment of the images. R.G.-R. thanks José Antonio Acosta Pulido for the encouragement to apply for the observing time of the E2 source and Ismael Pérez Fournón for his help with the analysis of the quasar's spectra. We thank Laura Magrini, the referee of this paper, for the detailed revision and suggestions that helped to significantly improve the article. The work was supported by the Spanish project AYA2012-35330.

\section{References}

Ahn, C. P., Alexandroff, R., Allende Prieto, C., et al. 2012, ApJS, 203, 21 Alexandroff, R., Strauss, M. A., Greene, J. E., et al. 2013, MNRAS, 435, 3306 Annibali, F., Tosi, M., Romano, D., et al. 2017, ApJ, 843, 20

Arnaboldi, M. 2012, in IAU Symp., 283, 267

Arnaboldi, M., Longobardi, A., \& Gerhard, O. 2016, in The General Assembly of Galaxy Halos: Structure, Origin and Evolution, eds. A. Bragaglia, M. Arnaboldi, M. Rejkuba, \& D. Romano, IAU Symp., 317, 69

Badenes, C., Maoz, D., \& Ciardullo, R. 2015, ApJ, 804, L25

Barker, M. K., Sarajedini, A., Geisler, D., Harding, P., \& Schommer, R. 2007a, AJ, 133, 1125
Barker, M. K., Sarajedini, A., Geisler, D., Harding, P., \& Schommer, R. 2007b, AJ, 133, 1138

Barker, M. K., Ferguson, A. M. N., Cole, A. A., et al. 2011, MNRAS, 410, 504

Bernard, E. J., Ferguson, A. M. N., \& Barker, M. K. 2012, in Galactic Archaeology: Near-Field Cosmology and the Formation of the Milky Way, eds. W. Aoki, M. Ishigaki, T. Suda, T. Tsujimoto, \& N. Arimoto, ASP Conf. Ser., 458,315

Bertin, E. 2006, in Astronomical Data Analysis Software and Systems XV, eds. C. Gabriel, C. Arviset, D. Ponz, \& S. Enrique, ASP Conf. Ser., 351, 112 Bertin, E., \& Arnouts, S. 1996, A\&AS, 117, 393

Bresolin, F., Ryan-Weber, E., Kennicutt, R. C., \& Goddard, Q. 2009, ApJ, 695 580

Buzzoni, A., Arnaboldi, M., \& Corradi, R. L. M. 2006, MNRAS, 368, 877

Cardelli, J. A., Clayton, G. C., \& Mathis, J. S. 1989, ApJ, 345, 245

Chandar, R., Bianchi, L., Ford, H. C., \& Sarajedini, A. 2002, ApJ, 564, 712

Ciardullo, R. 2013, in Advancing the Physics of Cosmic Distances, ed. R. de Grijs, IAU Symp., 289, 247

Ciardullo, R., Feldmeier, J. J., Jacoby, G. H., et al. 2002, ApJ, 577, 31

Ciardullo, R., Durrell, P. R., Laychak, M. B., et al. 2004, ApJ, 614, 167

Cioni, M.-R. L., Irwin, M., Ferguson, A. M. N., et al. 2008, A\&A, 487, 131

Cockcroft, R., McConnachie, A. W., Harris, W. E., et al. 2013, MNRAS, 428, 1248

Corradi, R. L. M., Magrini, L., Greimel, R., et al. 2005, A\&A, 431, 555

Corradi, R. L. M., Kwitter, K. B., Balick, B., Henry, R. B. C., \& Hensley, K. 2015, ApJ, 807, 181

Drew, J. E., Greimel, R., Irwin, M. J., et al. 2005, MNRAS, 362, 753

Ferguson, A., Irwin2, M., Chapman, S., et al. 2007, Astrophys. Space Sci. Proc., 3, 239

Freedman, W. L., Madore, B. F., Gibson, B. K., et al. 2001, ApJ, 553, 47

Gerhard, O., Arnaboldi, M., Freeman, K. C., et al. 2007, A\&A, 468, 815

Hainline, K. N., Shapley, A. E., Greene, J. E., \& Steidel, C. C. 2011, ApJ, 733, 31

Hodge, P. W., Balsley, J., Wyder, T. K., \& Skelton, B. P. 1999, VizieR Online Data Catalog: J/PASP/111/685 
Ibata, R., Martin, N. F., Irwin, M., et al. 2007, ApJ, 671, 1591

Irwin, M., \& Lewis, J. 2001, New Astron. Rev., 45, 105

Jacoby, G. H. 1980, ApJS, 42, 1

Jacoby, G. H. 1989, ApJ, 339, 39

Longobardi, A., Arnaboldi, M., Gerhard, O., \& Hanuschik, R. 2015, A\&A, 579, A135

Magrini, L., \& Gonçalves, D. R. 2009, MNRAS, 398, 280

Magrini, L., Corradi, R. L. M., Mampaso, A., \& Perinotto, M. 2000, A\&A, 355 713

Magrini, L., Corradi, R. L. M., Walton, N. A., et al. 2002, A\&A, 386, 869

Magrini, L., Corradi, R. L. M., Greimel, R., et al. 2003, A\&A, 407, 51

Magrini, L., Stanghellini, L., \& Villaver, E. 2009, ApJ, 696, 729

Magrini, L., Coccato, L., Stanghellini, L., Casasola, V., \& Galli, D. 2016, A\&A, 588, A91

Makarov, D., Prugniel, P., Terekhova, N., Courtois, H., \& Vauglin, I. 2014, A\&A 570, A13

Marmo, C., \& Bertin, E. 2008, in Astronomical Data Analysis Software and Systems XVII, eds. R. W. Argyle, P. S. Bunclark, \& J. R. Lewis, ASP Conf. Ser., 394, 619

McConnachie, A. W., Irwin, M. J., Ibata, R. A., et al. 2009, Nature, 461, 66

McConnachie, A. W., Ferguson, A. M. N., Irwin, M. J., et al. 2010, ApJ, 723, 1038

McMonigal, B., Lewis, G. F., Brewer, B. J., et al. 2016, MNRAS, 461, 4374

Merrett, H. R., Merrifield, M. R., Douglas, N. G., et al. 2006, MNRAS, 369, 120
Mikołajewska, J., Shara, M. M., Caldwell, N., Iłkiewicz, K., \& Zurek, D. 2017, MNRAS, 465, 1699

Minniti, D., \& Zijlstra, A. A. 1997, AJ, 114, 147

Oke, J. B., \& Gunn, J. E. 1983, ApJ, 266, 713

Osterbrock, D. E., \& Ferland, G. J. 2006, Astrophysics of Gaseous Nebulae and Active Galactic Nuclei (CA: University Science Books)

Patel, E., Besla, G., \& Sohn, S. T. 2017, MNRAS, 464, 3825

Pritzl, B. J., Olszewski, E. W., Saha, A., Venn, K. A., \& Skillman, E. D. 2011, AJ, 142, 198

Renzini, A., \& Buzzoni, A. 1986, in Spectral Evolution of Galaxies, eds C. Chiosi, \& A. Renzini, Astrophysics and Space Science Library, 122, 195-231

Rogstad, D. H., Wright, M. C. H., \& Lockhart, I. A. 1976, ApJ, 204, 703

Sarajedini, A., Barker, M. K., Geisler, D., Harding, P., \& Schommer, R. 2006, AJ, 132, 1361

Schönberner, D., Jacob, R., Steffen, M., \& Sandin, C. 2007, A\&A, 473, 467

Stetson, P. B. 1987, PASP, 99, 191

Stone, R. P. S. 1977, ApJ, 218, 767

van Dokkum, P. G. 2001, PASP, 113, 1420

Viironen, K., Delgado-Inglada, G., Mampaso, A., Magrini, L., \& Corradi, R. L. M. 2007, MNRAS, 381, 1719

Wang, F., Wu, X.-B., Fan, X., et al. 2016, ApJ, 819, 24

Wyder, T. K., Hodge, P. W., \& Skelton, B. P. 1997, PASP, 109, 927

Yang, S.-C., Sarajedini, A., Holtzman, J. A., \& Garnett, D. R. 2010, ApJ, 724 799 


\section{Appendix A: Spectra of a type II QSO candidate}

A $3 \times 2400 \mathrm{~s}$ spectrum of the [OIII] excess source E2 in Table 5 was taken on August 14 and September 2, 2017 with the same set-up as described in Sect. 2. The standard star HD 19445 (Oke \& Gunn 1983) was used for flux calibration. The spectrum is shown in Fig. A.1. Despite the faintness of the source, the characteristic lines of a typical active galactic nucleus (AGN; Hainline et al. 2011), at redshift $z \sim 3.12$, are recognized. The rest frame and observed wavelength, corresponding ion, and measured flux are tabulated in Table A.1. The FWHM of the critical Ly $\alpha$ and CIV lines are included. Type II AGNs are usually identified by their narrow emission lines $\left(F W H M<2000 \mathrm{~km} \mathrm{~s}^{-1}\right)$ and sources as faint as this object are barely detected (Alexandroff et al. 2013).

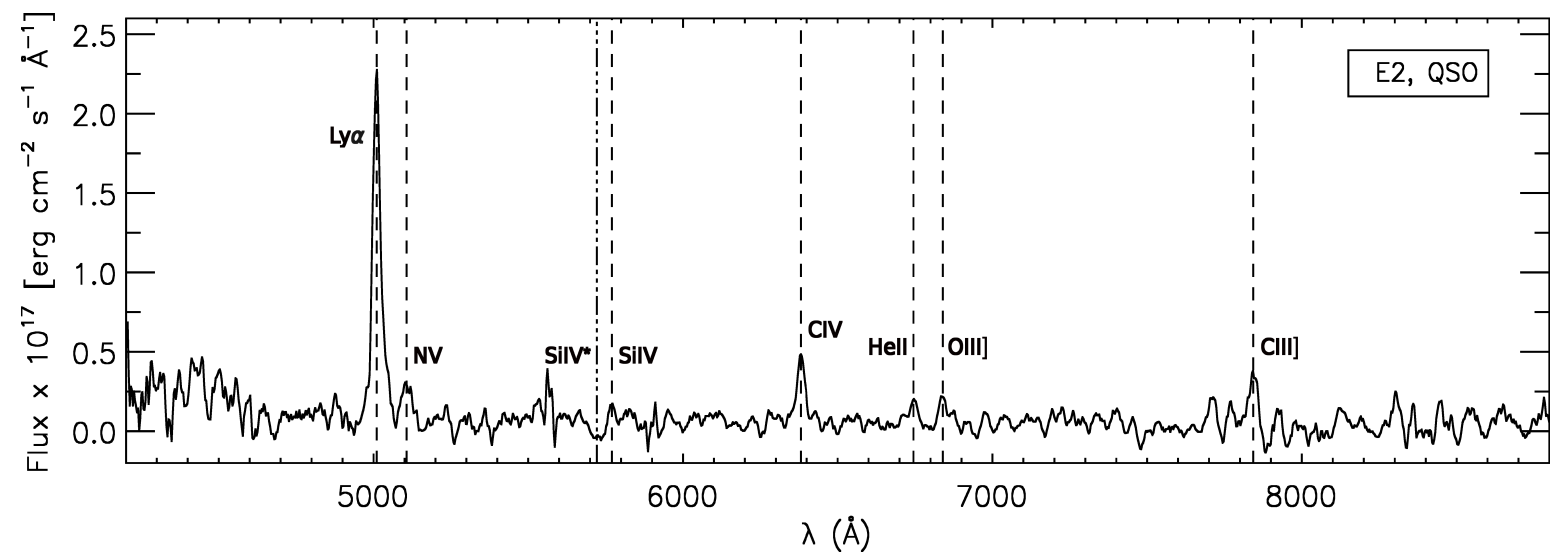

Fig. A.1. Spectrum of the [OIII] excess source E2, a type II QSO candidate.

Table A.1. Emission and absorption detected features.

\begin{tabular}{lcccc}
\hline \hline Ion & $\begin{array}{c}\lambda_{\text {rest }} \\
(\AA)\end{array}$ & $\begin{array}{c}\lambda_{\text {obs }} \\
(\AA)\end{array}$ & $\begin{array}{c}\text { FWHM } \\
\left(\mathrm{km} \mathrm{s}^{-1}\right)\end{array}$ & $\begin{array}{c}\text { Measured Flux } \\
\left(10^{-17} \mathrm{erg} \mathrm{s}^{-1} \mathrm{~cm}^{-2}\right)\end{array}$ \\
\hline Ly $\alpha$ & 1215.7 & $5010.1 \pm 0.2$ & $1848 \pm 70$ & $69 \pm 4$ \\
NV & $1238.8,1242.8(1240.15)$ & $5107.3 \pm 2.3$ & - & $8.2 \pm 1.1$ \\
SiIV & 1393.7 & $5723.0 \pm 4.1$ & - & $-8.2 \pm 1.0$ \\
SiIV & 1042.8 & $5770.9 \pm 4.7$ & - & $2.8 \pm 1.6$ \\
CIV & $1548.2,1550.8(1549.06)$ & $6381.3 \pm 1.4$ & $1373 \pm 61$ & $12.9 \pm 1.2$ \\
HeII & 1640.4 & $6747.3 \pm 5.3$ & - & $4.9 \pm 0.9$ \\
OIII & 1660.8 & $6840.4 \pm 2.7$ & - & $6.0 \pm 0.8$ \\
CIII & $1906.8,1908.7$ & $7843.6 \pm 1.4$ & - & $11.3 \pm 1.4$ \\
\hline
\end{tabular}

Notes. ${ }^{(a)}$ Absorption. 